\title{
Effects and Mechanism of SDF-1a/CXCR4 pathway on cerebral neurogenesis and angiogenesis in recovery phase after intracerebral hemorrhage in rats
}

\author{
Tian Cao ${ }^{1}$, Bo $\mathrm{Wu}^{1}$, Di Zhong ${ }^{1}$, Yuyin Yan ${ }^{1}$. \\ Sichuan University, the Stroke Clinical Research Unit, Department of Neurology,West \\ China Hospital, Chengdu, China.
}

\section{Background and Aims}

Central nervous system constitutively express SDF$1 /$ CXCR4 which promotes the migration of neural precursor cells, proliferation, differentiation of neurons, and regulates neuronal loop formation. Following intracerebral hemorrhage $(\mathrm{ICH})$, whether SDF-1a/CXCR4 regulates or participates in neurovascular regeneration is unclear, as are the pattern recognition receptors and signaling pathways involved.

\section{Methods}

$\mathrm{ICH}$ was induced by injection of collagenase in Sprague-Dawley rats. Then the SDF- $\alpha$ /CXCR4 axis inhibitor AMD3100 were injected intraperitoneally every day in the following 14 days. Injection of saline were set as a control group. A sham group was treated with saline instead of collagenase and then with saline in the following 14 days. Observe all animal's modified neurological severity scores (mNSS) at 1, 3, 7, 10, and 14 day after $\mathrm{ICH}$. Expression of the following proteins was measured by western blot: SDF-1a, CXCR4, BDNF, VEGF, and pERK. The number of cells positive for 5-bromo-2deoxyuridine or doublecortin was determined by immunohistochemistry.

\section{Results}

Levels of SDF-1 $\alpha$, CXCR4, BDNF, VEGF, and p-ERK were significantly higher 14 days after $\mathrm{ICH}$ than at baseline, as were the numbers of 5-bromo-2deoxyuridine- or doublecortin-positive cells. Daily administration of AMD3100, which inhibited SDFa/CXCR4 axis, reduced the recovery of neurological function. Administration of AMD3100 significantly reduced all $\mathrm{ICH}$-induced increases of Brdu+/DCX+/CD31+ cells increases.

\section{Conclusion}

SDF-1 $\alpha / C X C R 4$ upregulation accompanied VEGF, p-ERK, and BDNF increases around $\mathrm{ICH}$ lesions. Inhibition of the binding of SDF-1 $\alpha$ and CXCR4 impede neurovascular regeneration, suggesting that SDF-1 $\alpha$ /CXCR4 involved in neurovascular unit repair.
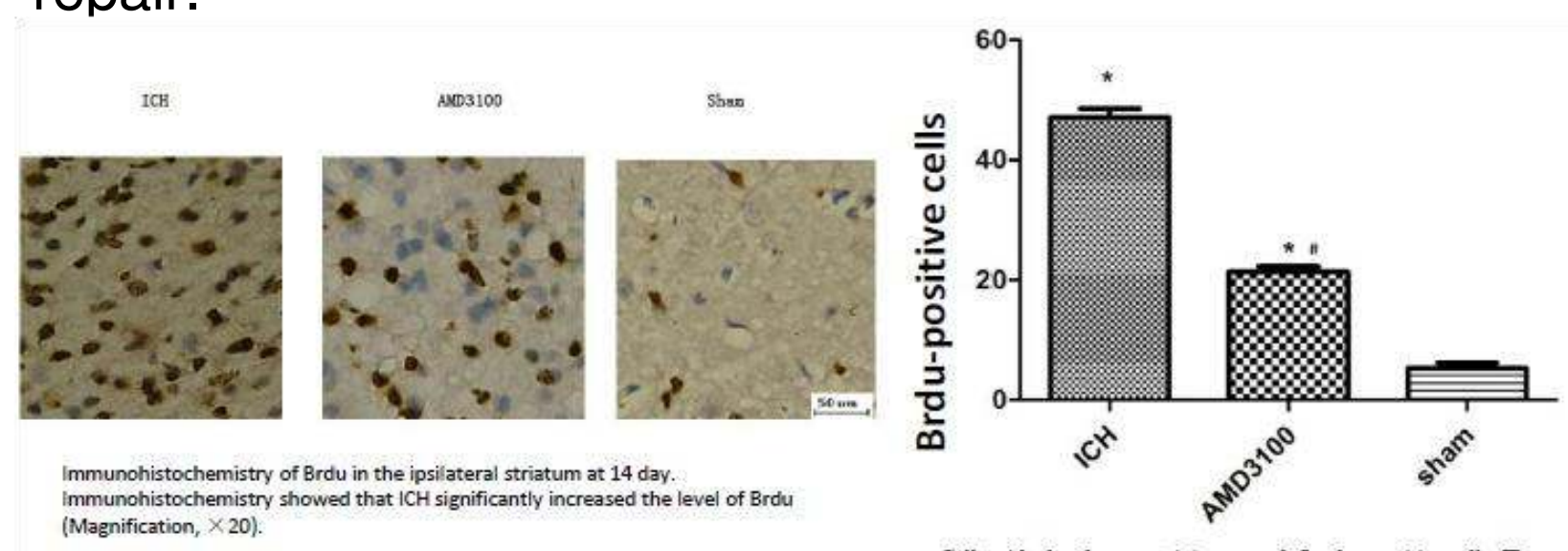

$\mathrm{ICH}$
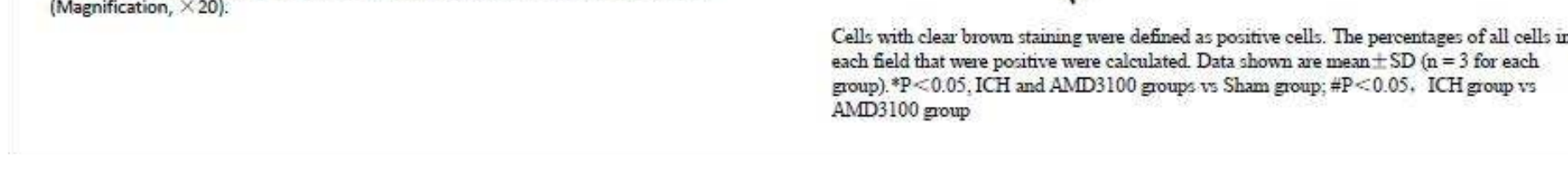

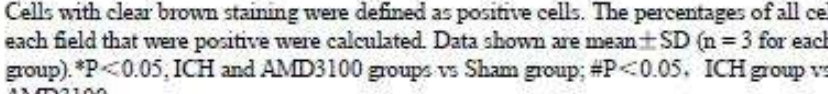

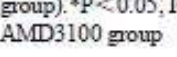
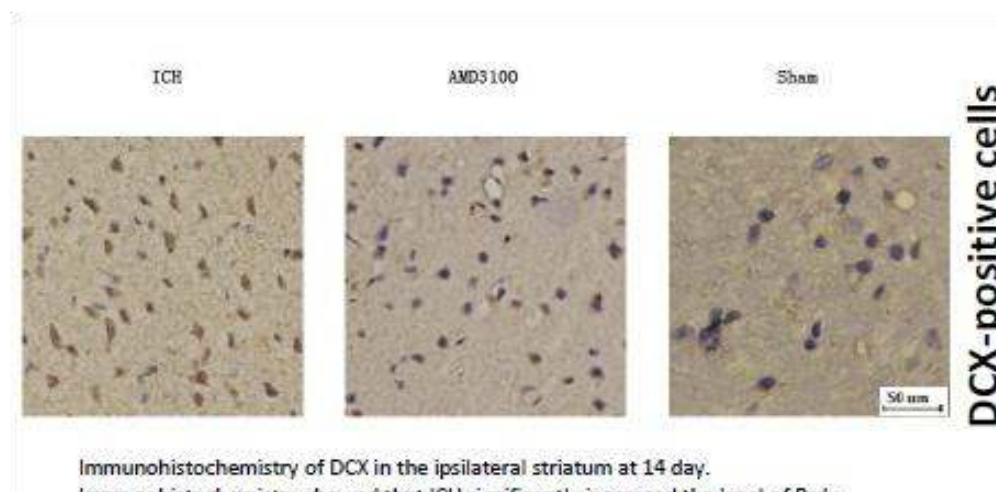

Immunohistochemistry of DCX in the ipsilateral striatum at $14 \mathrm{~d}$
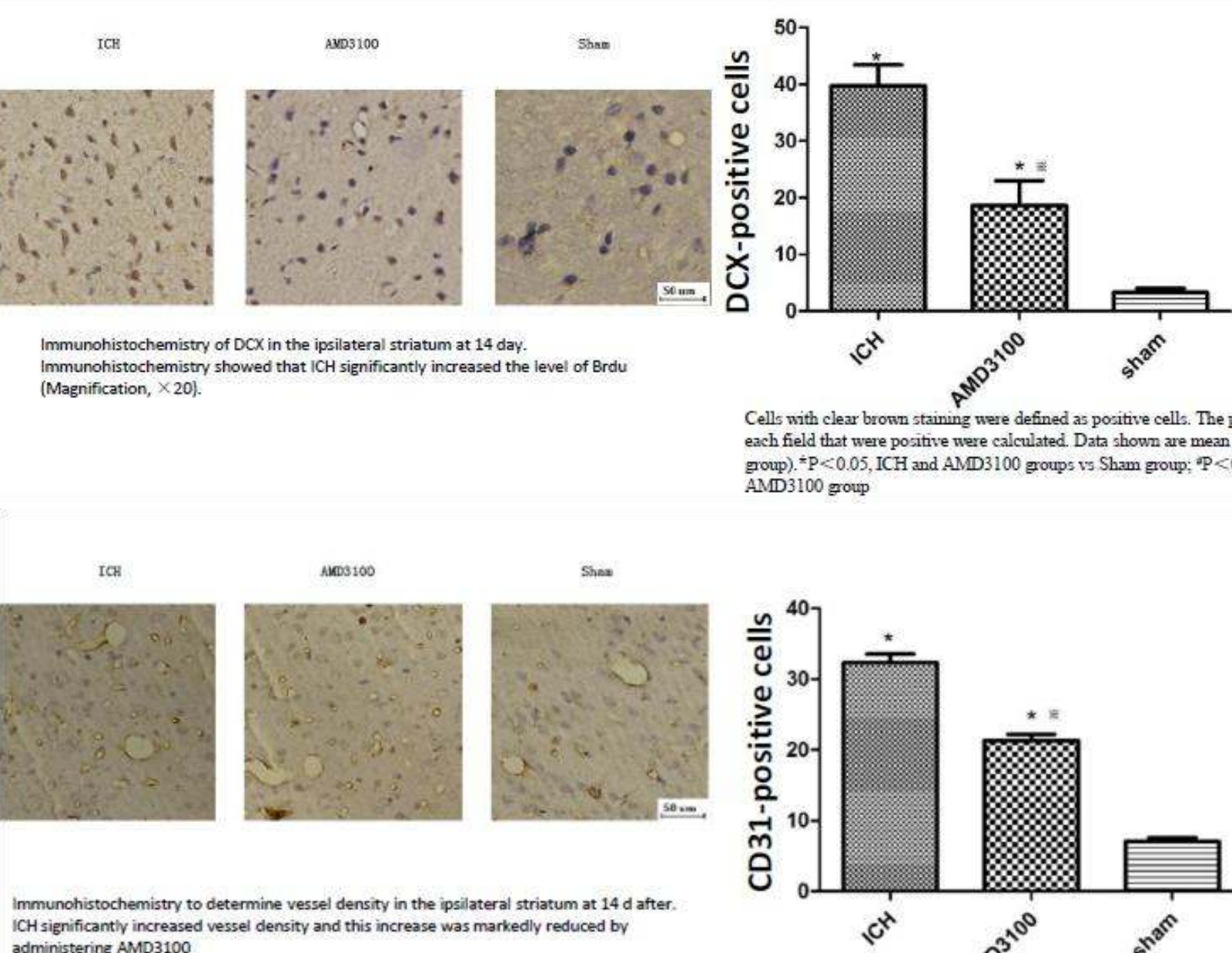

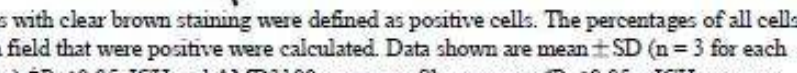

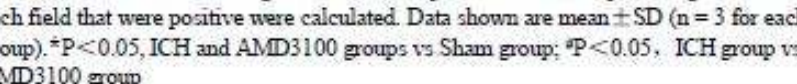
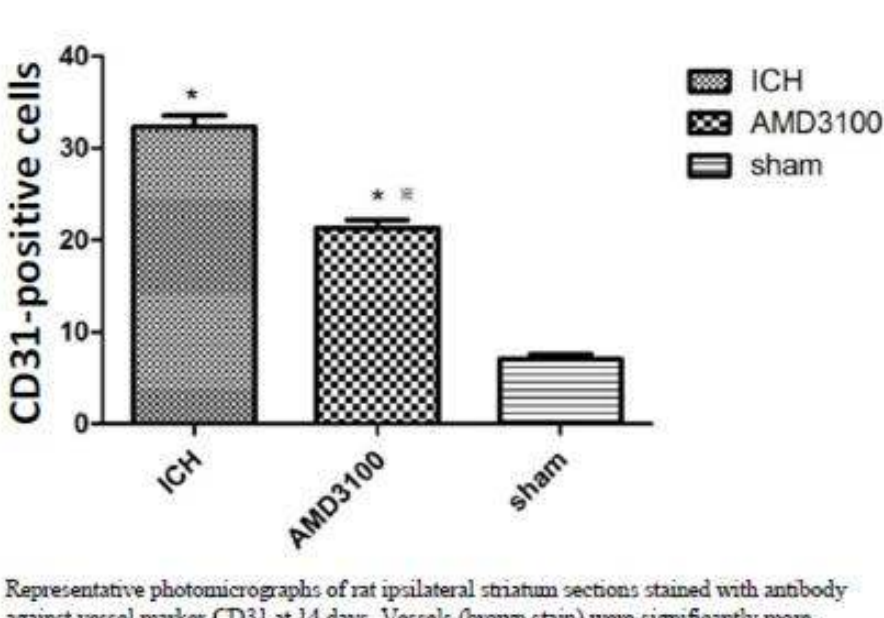

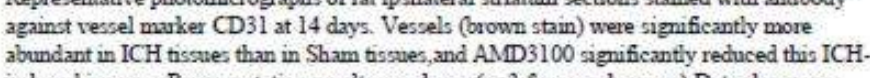

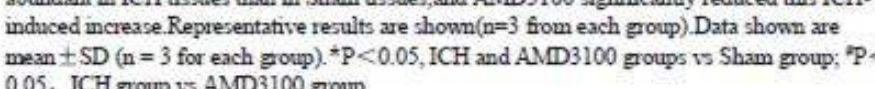

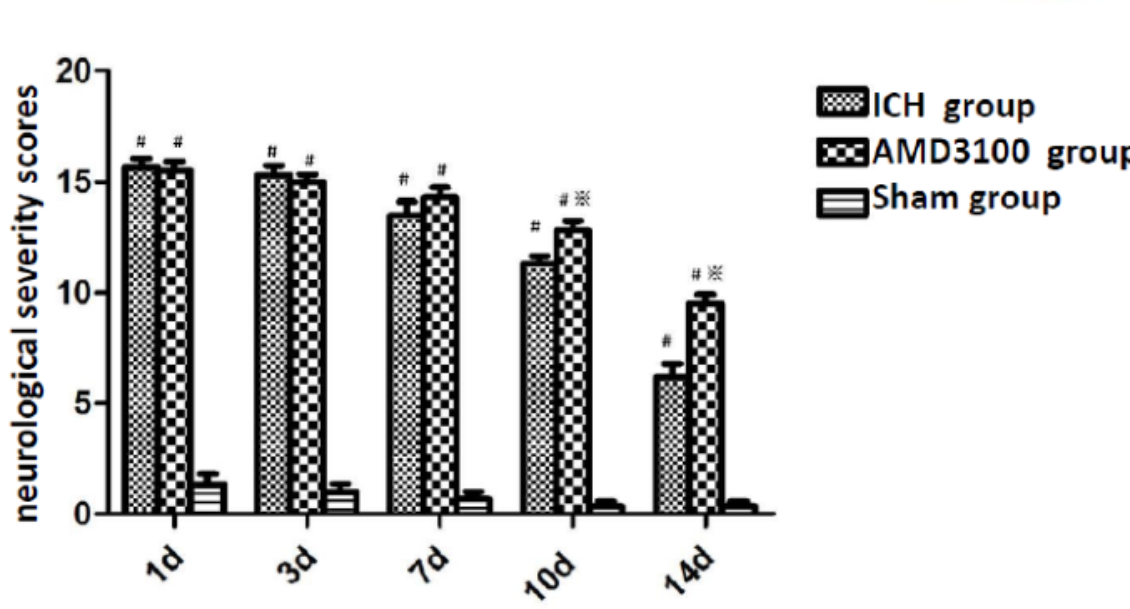

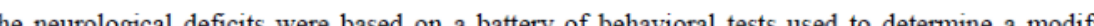

neurological severity score (mNSS). Neurological function was lower in ICH animals than in Sham animals based on behavioral tests. Treatment with AMD3100 after ICH inhibited this recovery. Overall across all tasks are shown as mean \pm

Copyright $@ 2017$ Corresponding author at: Stroke Clinical Research Unit, Department of Neurology, West China Hospital,Sichuan University,E-mail address: dragonwbtxf@hotmail.com (B. Wu). 